\title{
iWordNet: A New Approach to Cognitive Science and Artificial Intelligence
}

\author{
Mark Chang ${ }^{1}$ and Monica Chang ${ }^{2}$ \\ ${ }^{1}$ Boston University, 801 Massachusetts Ave, Boston, MA, USA \\ ${ }^{2}$ Carnegie Mellon University, 5000 Forbes Ave, Pittsburgh, PA 15213, USA \\ Correspondence should be addressed to Mark Chang; mychang@bu.edu
}

Received 5 April 2017; Revised 18 July 2017; Accepted 28 August 2017; Published 11 October 2017

Academic Editor: António Dourado Pereira Correia

Copyright (c) 2017 Mark Chang and Monica Chang. This is an open access article distributed under the Creative Commons Attribution License, which permits unrestricted use, distribution, and reproduction in any medium, provided the original work is properly cited.

\begin{abstract}
One of the main challenges in artificial intelligence or computational linguistics is understanding the meaning of a word or concept. We argue that the connotation of the term "understanding," or the meaning of the word "meaning," is merely a word mapping game due to unavoidable circular definitions. These circular definitions arise when an individual defines a concept, the concepts in its definition, and so on, eventually forming a personalized network of concepts, which we call an iWordNet. Such an iWordNet serves as an external representation of an individual's knowledge and state of mind at the time of the network construction. As a result, "understanding" and knowledge can be regarded as a calculable statistical property of iWordNet topology. We will discuss the construction and analysis of the iWordNet, as well as the proposed "Path of Understanding" in an iWordNet that characterizes an individual's understanding of a complex concept such as a written passage. In our pilot study of 20 subjects we used a regression model to demonstrate that the topological properties of an individual's iWordNet are related to his IQ score, a relationship that suggests iWordNets as a potential new methodology to studying cognitive science and artificial intelligence.
\end{abstract}

\section{Introduction}

Cognitive science, as an interdisciplinary study of mind and intelligence, embraces philosophy, psychology, artificial intelligence, neuroscience, linguistics, and sociology. As cognitive science continues to evolve, artificial intelligent approaches such as statistical learning and network science are becoming increasingly attractive and relevant to researchers in cognitive science and artificial intelligence. Recent work [16] demonstrates that statistical learning is one of the most deeply explored phenomena in the field of cognitive science. Noticeably, the increasing utilization of network science with statistical learning [7-9] makes network-based approaches a robust tool in cognitive science and computational linguistics $[10,11]$. Network science has been successfully applied to several pressing issues: feature biases in early word learning [12], semantic concepts [13-17], grammatical relationships [18], spatial learning in human navigation [19], structural and functional brain connections in relationship to various cognitive capacities [20-23], and scene perception studies such as texture and shape discrimination [24-26]. Perhaps the field that has most broadly adopted network-based approaches in cognitive science is computational linguistics [27-33].

Despite these numerous advances, the integration of network science and cognitive science has not been carried out in the way that we hoped for. The research tends to focus either on (i) the description of networks derived from the sensory world, or (ii) the mechanisms by which the human brain engages with the sensory world, with little communication between these two areas, as Karuza et al. [34] commented in their recent review paper, "Influences of Network Topology on Human Learning." The authors pointed out: "... a dominant approach to human learning has focused on microlevel patterns, often the pairwise relationships between the constituents of sensory input. ... Currently, many quantitative approaches to learning hinge on sensitivity to local statistics such as cooccurrence frequencies and transitional probabilities between adjacent elements." They urge us to examine how internal complex system dynamics gives 
rise to learning, how acquired knowledge might be reflected in observable topological patterns in the human brain, and how micro- and macrolevel brain dynamics support learning.

It is undeniable that our lives today are intimately tied to various external networks, whether it is friends, transportation, or social media networks. The collective analyses of social media networks have offered unprecedented insight into how humans transmit information and interact with one another [34-37]. To use network science methodology to study the mind and cognitive development, knowledge needs to be expressed as a network of concepts, which reflects one's state of mind and collective intelligence.

Different from human neural networks, which are commonly studied in cognitive science, we propose a method of constructing individualized networks of concepts, called iWordNets, which reflect an individual's knowledge or state of mind at the time of construction. By studying the topology of the iWordNet, we can learn how the person's cognition was developed, how it differs in comparison to others, and why people experience different cognitive developments. This information can further provide potential insights into improving teaching, learning, educational evaluations, and patient treatments in clinics.

An iWordNet can be constructed through continuously asking a subject to explain concepts, one after another, recursively. We will first examine the connotation of the term "understanding" and prove that the meaning of "understanding" is essentially a word mapping game and that a word is just a placeholder. Based on this view, we postulate that there are relationships between the topology of an individual's iWordNet and his overall knowledge or IQ and use a small pilot study to test the hypothesis. Encouraged by the initial positive findings between IQ and iWordNet topology, we further describe how to use iWordNets to study an individual's understanding of a concept, sentence, or paragraph using the so-called Path of Understanding (PoU). We quantify the meaning of a string of words using topological characteristics (such as degree, centrality) along the PoU to explain how people might acquire new knowledge. Thus, the iWordNet as an artificial intelligent approach potentially provides a new, noninvasive, low-cost way to study the brain, mind, and cognitive science.

\section{The Connotation of Understanding}

Knowledge is a collection and organization of concepts and consists of many pieces of individual "understanding." However, we rarely question the understanding of a concept. What does the term "understanding" mean? Does each person have a unique understanding of a concept and to what extent might they vary? What role does understanding play in our communication?

We can say that a library, a collection of books, a research paper, the news, and so forth are bodies of knowledge or collections of concepts. A library is a relatively simple organized repository of concepts and so is a social media network. In a dictionary, words are defined by other words, which are further defined by other words, and so on. If we connect these words using lines to reflect their direct relationships, we form a network of words, which can be considered as knowledge external to any individuals. Similar to how we would construct a dictionary network, a person can explain a concept by other concepts, which are further explained by other concepts, and so on. If we connect a concept with the concepts directly used in the explanation, we form a network of concepts (Figure 1). This type of networks reflects the state of mind of the person, making it a potential tool for studying individual's knowledge, learning process, abilities, and so forth. Such a network is an external representation of an individual's knowledge. We call such a network an iWordNet for lack of a better term. The letter " $i$ " in "iWordNet" reflects the individualistic nature of the network. An iWordNet will evolve over time and vary from individual to individual. Therefore, studying iWordNets can help us to learn about the cognitive development of an individual and explain the differences among different groups of individuals. If an iWordNet is constructed by all the people in an organization, it measures or reflects the collective intelligence of the organization. As a result, an iWordNet can be used to determine both an individual's and organization's behaviors and predict their successes and failures. Before we can elaborate all these, however, we need to define "understanding" or "meaning" as a statistical problem surrounding iWordNets. We will start with the investigation of the connotation of understanding.

A concept is defined by other concepts, which are further defined by other concepts. Since one has a limited number of concepts, he will ultimately have an iWordNet in which all concepts have circular definitions or words that cannot be defined by other concepts. Furthermore, the meaning of each word is different for different individuals and shifts constantly as time goes by. Most of the time people think they understand each other when they establish a mapping (or agreement) between what they perceive. It is synonymous with hearing the phrase: "I know what you are talking about" or "I understand what you mean." Although one's perceived understanding and one's actual understanding can be different, it does not matter as long as one thinks the other understands, and vice versa. That is, the meaning of a word has to be defined in terms of the individual and is nothing but mapping in an iWordNet that is defined by the topology of the iWordNet. In this sense, meaning is meaningless, an idea which we call the Paradox of Understanding.

If you look in the online Webster dictionary, you will find that confusion means perplexity, perplexity means bewilderment, bewilderment means incomprehension, and incomprehension means confusion, which turns out to be a cyclic network with many loops or circular definitions.

Because of the circular definitions, the concept in a node is insignificant and can be replaced with anything else: a symbol in a different language, or simply a differently shaped node. Consequently, the overall level of knowledge of a person can be characterized by the topology of the iWordNet. In our small pilot study, we will show you how the topology of iWordNets correlates to IQ score. 


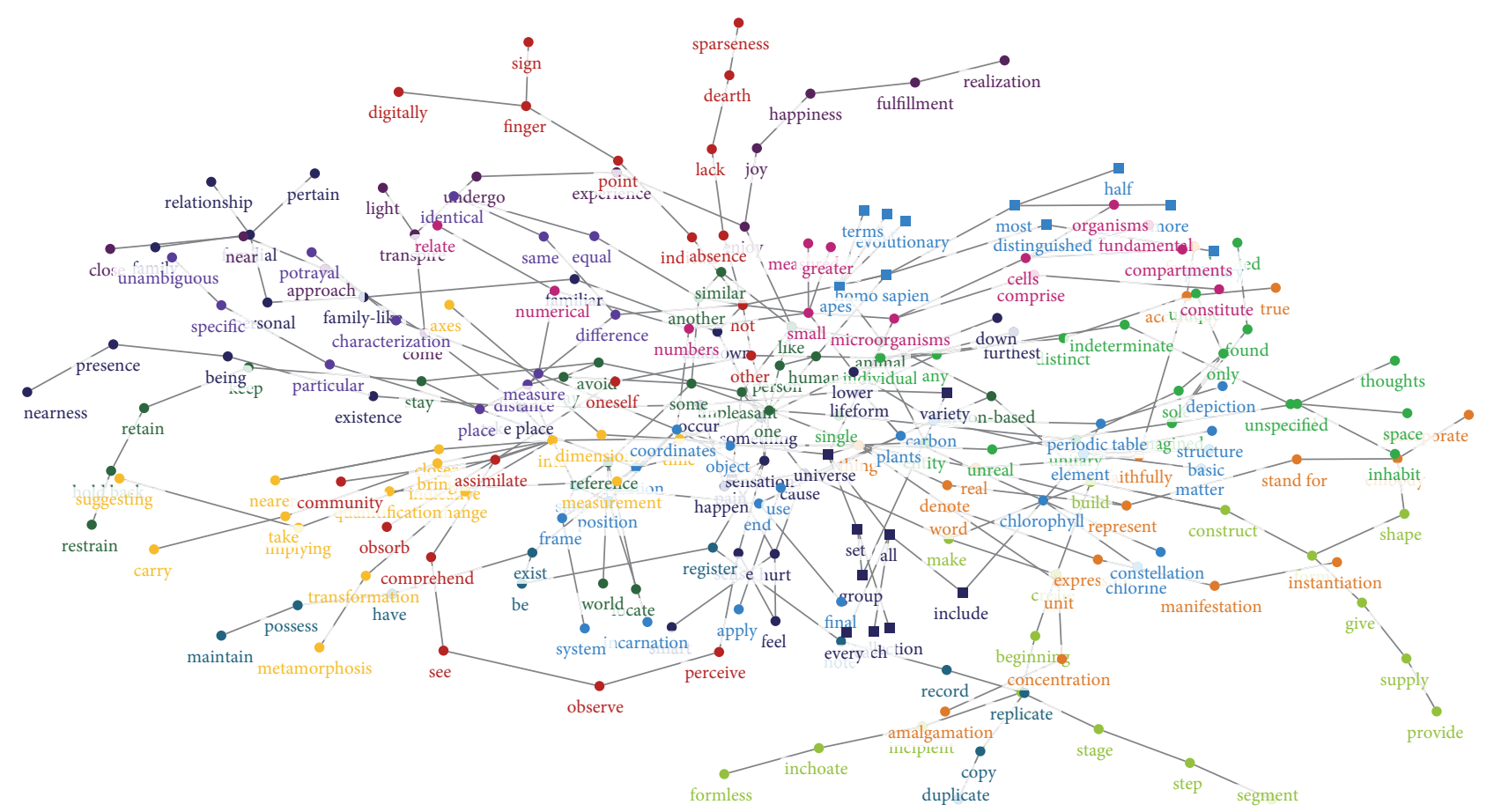

Figure 1: An example of a knowledge network.

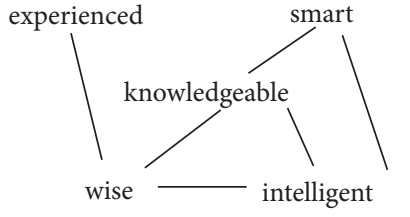

Figure 2: A simple iWordNet.

\section{The iWordNet}

An iWordNet is not a network of synonyms. Using concepts to explain other concepts in an iWordNet implies the necessity of understanding the concepts before understanding the concept in question. For instance, if you use "knowledgeable" to explain "smart," even though their meanings may be different, you need to know the meaning of "knowledgeable" to understand "smart." Moreover, in an iWordNet, the items at the vertices (nodes) of the network are not limited to words; they can include phrases, long text strings, sentences, symbols, pictures representing activities or events, music clips, video clips, and other relevant items. The items that are directly connected do not have to be related in the sense that one explains the other. Other relationships may be equally valid. A causal relationship, for example, could be how "hungry" makes one think of "food." Figure 2 is a small portion of an iWordNet in a graphic form, whereas Table 1 is the same portion of the iWordNet in a table form.

When the edges in the networks are directionless, the networks are undirected, dependent on whether the edges are directional or not. For example, we can use an arrow to
TABLE 1: iWordNet in table format.

\begin{tabular}{lc}
\hline smart & intelligent \\
smart & knowledgeable \\
intelligent & wise \\
intelligent & knowledgeable \\
wise & knowledgeable \\
wise & experienced \\
\hline
\end{tabular}

represent a causal relationship. We can also put a weight on each edge to indicate the strength of the relationship or other properties, such as in the Petri Net [38-40] in biological and electronic networks.

Unlike nodes in neural network models in cognitive neuropsychology, those in iWordNets have no meaning in terms of physical location or physical distance with other nodes. Neural networks focus on information processes, while iWordNets deal with both knowledge itself and learning mechanisms. iWordNets are also different from artificial neural networks. An ANN is a learning model, while an iWordNet is a representation of individual knowledge on which learning mechanisms can be built.

WordNet ${ }^{\circledR}$ by Princeton University is a large lexical database of English. Nouns, verbs, adjectives, and adverbs are grouped into sets of cognitive synonyms (synsets), each expressing a distinct concept. WordNet ${ }^{\circledR}$ focuses on word usage. Similarly, ConceptNet by MIT is a semantic network containing concepts that can be used for a machine to "understand" texts written by humans. A fundamental difference between 
iWordNet and these two networks is that WordNet ${ }^{\circledR}$ and ConceptNet are exhaustive collections of words, concepts, and their usage and thus contain no information about any individual, whereas iWordNets are individualized and can be used to study an individual's cognitive development. This will become much clearer when we discuss the Path of Understanding below.

3.1. The Concept of Constructing an iWordNet. An iWordNet can be constructed in many different forms, including a graphical network form, a table-like form, and a database-like form. Here we describe a simple way used in our pilot study.

(1) Pick a starting word (phrase, concept, picture, or object) and ask, face-to-face or online, a subject to explain the meaning of the word. Record the list of words used by the user to define the starting word.

(2) Make a connection between the word to be explained (i.e., the starting word) and each word used in the explanation. Such a connection can be a directed segment (an arrow) or an undirected segment (a line). The connection between the paired words can also be recorded in a table form by entering the two words in two different columns of the same row.

(3) Recursively ask the subject to explain the words used in the explanation, creating more connections or edges. Continue this process until an iWordNet is constructed. In the process, some concepts or words used previously are expected to be used again, forming circular definitions. See the example in Figure 2. The criterion for the completion of an iWordNet can be subjective. In our pilot study, for simplicity, we stopped asking questions when the diameter of an iWordNet reached 10.

There are different methods for creating an iWordNet, digitally or by hand: we can use simple questions, computer games, and so forth. Concepts can be expressed by different languages, symbols, pictures, music clips, and any other objects, since a child acquires a concept often before he/she understands a language or word. A network can be constructed all at once or piecewise over time.

3.2. Modeling Cognition with the iWordNet. The local topological properties of an iWordNet include vertices (nodes, words, etc.), degrees, clusters, betweenness centrality, closeness centrality, eigenvalue centrality, PageRank, geodesic distance, and "top items." The global topological properties include number of vertices, number of edges, number of components, number of clusters, clustering coefficient, mean geodesic distance, mean closeness centrality, mean closeness centrality, number of unique edges, graph density, and any variables derived from these variables.

Modeling cognitive, emotional, and personality measurements with iWordNet topological properties can be done with regressions or other statistical modeling techniques. Other supervised and unsupervised methods in statistical learning can also be used.

\section{The Pilot Study}

As part of the pilot study, we were interested in how one's IQ and iWordNet are related. To pursue the answers, we obtained IQ scores and iWordNets from 20 male and female subjects with an age range of 16 to 60 years.

The IQ test we employed was designed to measure abilities in short-term memory, analytical thinking, abstract problem solving, mathematical ability, and spatial recognition (http://www.seemypersonality.com:80/IQ-Test, retrieved October 13, 2014). Like all IQ tests, the test we used was not intended to measure the amount of knowledge the individual has, but rather the capacity of the individual to learn. We were not able to get the subjects' academic transcripts; otherwise, we would have used them in addition to the IQ scores.

The IQ test, in addition to an overall IQ measurement, includes left brain and right brain IQ components. Left brain IQ measures memory, word comprehension, logic, and numerical sequences. Right brain IQ measures perception, visual designs, spatial relations, and creativity.

To construct the iWordNets, we first told each individual to define the word "smart." We chose to use the word "smart" because we conjectured that if the starting word is related to IQ, it would help us to establish a relationship between them. After defining the first word, the subject was told to define the key words in its definition and, furthermore, the key words in the definitions of the definitions. The key words were chosen based on their importance in an individual's definition. For example, if a person defined "smart" as "the state of being intellectual or having knowledge," we would record the words "state," "being," "intellectual," "have," and "knowledge." The words and the key words in their definitions are considered to be the vertices of the network and are linked together with lines (edges) to form a network of words, that is, an iWordNet.

By analyzing the iWordNet for each individual, we obtained the topological properties, such as the degree, geodesic distance, and modularity, of the networks. Then, we modeled the relationships between network properties and IQ results using a linear model.

Materials. Human subjects, computer with Internet access, online IQ test, NodeXL Basic (http://nodexl.codeplex.com, retrieved 2014), Statistical Analysis Software, and large papers for sketching iWordNets.

\subsection{The Procedure for Constructing the iWordNet}

(1) Recruit subjects, obtain their consent forms, and schedule an interview for constructing his/her iWordNet (an interview may take from as little as 30 minutes to as much as multiple hours).

(2) Give the subject the IQ test to take online at his/her convenience. The test will take about 10-15 minutes to finish. Keep an electronic copy of each subject's IQ scores (overall, left brain, and right brain).

(3) In a data collection document, record the subject number and his/her IQ scores.

(4) For the iWordNets, start with the word "smart," and tell the subject to define it. Tell the subject to define the key words in their definition. 


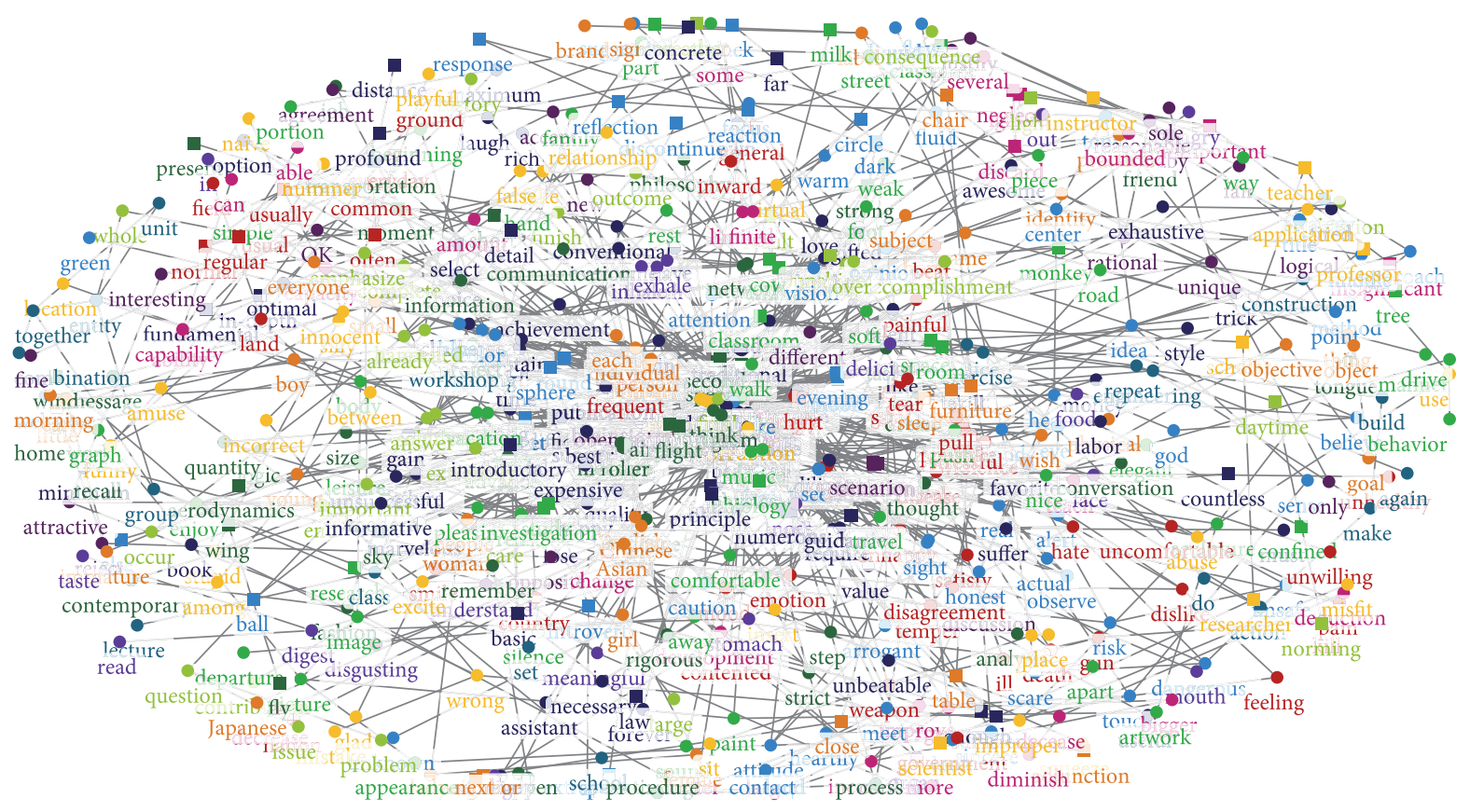

FIgURE 3: An iWordNet from the pilot study.

(5) As the subject speaks, record the key words in his/her definitions onto the large sheet of paper (creating a sort of "tree" diagram). Also enter the words and their defining words pairwise into NodeXL. After inputting the words for each subject into NodeXL, one will eventually be able to create an iWordNet for that individual.

(6) Continue with this defining process until the subject has reached up to 10 steps in chains of questioning from the starting word to the farthest word.

(7) Repeat steps (2)-(6) for all 20 subjects.

(8) Once steps (2)-(6) have been accomplished for all 20 subjects, use the tools in NodeXL [41] to analyze and generate topological properties for each iWordNet (centrality, vertices, geodesic distance, etc.).

(9) On the master spreadsheet, compile all the data, including the subject numbers, IQ scores, and values for the key topological properties of the iWordNets (Table 2).

4.2. The Global Topological Properties of the iWordNet. Because our pilot study was small, and the iWordNets were constructed in a relatively short amount of time, we do not want to overinterpret the findings, but we would like to point out a few interesting properties of the iWordNets.

The networks exhibit a clustered structure (ClausetNewman-Moore clustering algorithm) as indicated by the colors in the example of an iWordNet (Figure 3). The clustering implies that some words/concepts, as expected, are more loosely related than others. Modularity is designed to measure the strength of the division of a network into clusters.
Networks with high modularity have dense connections between the nodes within modules but sparse connections between nodes in different modules. The mean modularity of the iWordNets ranges from 0.53 to 0.76 , indicating a strong clustering structure. The minimum and maximum modularity values are -0.5 and 1 , respectively. A very low value of modularity for an iWordNet may indicate a person's confusion among different concepts, whereas an extremely high value may indicate a lack of creativity because minimally connected clusters may disable one's ability to make analogies across different fields, an essential part of creativity.

Among 20 iWordNets, although the number of vertices (words used) ranges widely from 35 to 600 , the mean geodesic (shortest distance by steps between two vertices) ranges from 4.4 to 8.8. Even the diameter (the largest geodesic distance) ranges from 12 to 18 , meaning any two words in any of the iWordNets can be reached with 12 to 18 steps. Thus, an iWordNet is clearly a small-world network. More interestingly, diameter, mean geodesic distance, and modularity do not necessarily increase or decrease as the number of vertices increases. The nature of the small-world network implies that concepts are much more closely related to each other than we expected.

Like many other small-world networks, such as electric power grids, metabolic networks, neural networks, voter networks, telephone call graphs, and social influence networks, iWordNets are robust against the random deletion of vertices. In other words, randomly forgetting some words does not affect the iWordNet or knowledge as significantly as it does for a random graph/network, where randomly deleting a vertex is likely to impact almost every vertex. While random networks are vulnerable to random perturbations, they cannot be targeted for catastrophic failure. Meanwhile, 
TABLE 2: Summary of key topological properties of 20 subjects.

\begin{tabular}{|c|c|c|c|c|c|c|c|c|c|c|}
\hline Subject & IQ & $\begin{array}{c}\text { Total } \\
\text { vertices }\end{array}$ & $\begin{array}{c}\text { Unique } \\
\text { edges }\end{array}$ & $\begin{array}{l}\text { Total } \\
\text { edges }\end{array}$ & Diameter & $\begin{array}{c}\text { Mean } \\
\text { geodesic }\end{array}$ & $\begin{array}{l}\text { Graph } \\
\text { density }\end{array}$ & Modularity & Mean degree & $\begin{array}{c}\text { Mean } \\
\text { betweenness } \\
\text { centrality }\end{array}$ \\
\hline 1 & 136 & 72 & 72 & 118 & 14 & 5.80 & 0.0344 & 0.5358 & 2.444 & 173.4 \\
\hline 2 & 144 & 184 & 199 & 233 & 18 & 7.41 & 0.0127 & 0.7048 & 2.135 & 590.5 \\
\hline 3 & 147 & 104 & 113 & 125 & 18 & 6.68 & 0.0222 & 0.7132 & 2.288 & 296.0 \\
\hline 4 & 89 & 605 & 981 & 1282 & 12 & 5.50 & 0.0060 & 0.6075 & 3.739 & 1360.4 \\
\hline 5 & 137 & 58 & 60 & 60 & 16 & 7.44 & 0.0363 & 0.7142 & 2.069 & 187.2 \\
\hline 6 & 145 & 35 & 33 & 35 & 14 & 6.30 & 0.0606 & 0.6278 & 2.000 & 90.5 \\
\hline 7 & 142 & 152 & 162 & 227 & 17 & 7.20 & 0.0163 & 0.6322 & 2.461 & 471.3 \\
\hline 8 & 144 & 151 & 148 & 170 & 19 & 8.59 & 0.0140 & 0.7573 & 2.093 & 568.1 \\
\hline 9 & 160 & 150 & 145 & 211 & 15 & 6.30 & 0.0160 & 0.6309 & 2.373 & 398.2 \\
\hline 10 & 156 & 202 & 322 & 412 & 12 & 4.38 & 0.0181 & 0.5516 & 3.634 & 342.3 \\
\hline 11 & 155 & 112 & 113 & 127 & 19 & 8.78 & 0.0193 & 0.7475 & 2.143 & 406.3 \\
\hline 12 & 155 & 121 & 161 & 191 & 12 & 4.70 & 0.0242 & 0.6030 & 2.909 & 217.3 \\
\hline 13 & 151 & 202 & 230 & 260 & 16 & 7.10 & 0.0121 & 0.7115 & 2.426 & 616.8 \\
\hline 14 & 146 & 105 & 83 & 172 & 16 & 7.33 & 0.0223 & 0.5309 & 2.324 & 332.7 \\
\hline 15 & 146 & 38 & 36 & 44 & 13 & 5.72 & 0.0569 & 0.5971 & 2.105 & 90.2 \\
\hline 16 & 146 & 72 & 76 & 86 & 14 & 6.44 & 0.0313 & 0.6591 & 2.25 & 196.3 \\
\hline 17 & 160 & 164 & 178 & 228 & 16 & 6.45 & 0.0151 & 0.6660 & 2.463 & 447.2 \\
\hline 18 & 154 & 152 & 178 & 230 & 12 & 5.65 & 0.0178 & 0.6367 & 2.684 & 354.3 \\
\hline 19 & 127 & 228 & 270 & 286 & 15 & 6.53 & 0.0107 & 0.7164 & 2.439 & 630.8 \\
\hline 20 & 160 & 55 & 57 & 61 & 13 & 6.08 & 0.0397 & 0.6660 & 2.145 & 140.1 \\
\hline
\end{tabular}

small-world networks are usually vulnerable to targeted attacks of hubs, but within the 20 iWordNets, there is only a maximum degree of 10 to 15 , so no significant hubs exist. Of course when an iWordNet gets larger with more time for the construction, there could be more significant hubs.

As expected, the data indicate that the total number of unique edges and the mean betweenness centrality increase linearly as the total number of vertices increases. However, the graphic density and mean eigenvector centrality decrease exponentially as the total number of vertices increases.

\subsection{Statistical Modeling of IQ with Global iWordNet Prop-} erties. We have described the topological properties of the iWordNet. Now we will investigate how these properties are related to knowledge or IQ. We initially included 9 topological variables of iWordNets in the IQ modeling. We used linear regression with backward-elimination methods and a staying $p$ value less than 0.1 . Because the time of the experiment for each subject was not fully controlled (most subjects took about one hour of questioning to their iWordNet, but some took up to several hours) and because the betweenness centrality increases as the time increases, we need to include variables that are stable as the experiment time changes to adjust for the effect. We identified stabilized centrality such as a variable, defined as the betweenness centrality divided by the total number of edges. Under the same logic, we also included another "stable factor," stabilized density, defined as graph density divided by the number vertices.

The final model includes statistically significant factors, MD (mean degree), M (modularity), NE (total number of edges), UE (number of unique edges), GD (graphic density), SD (stabilized density), and SC (stabilized centrality) with associated $p$ values, 0.0199, 0.0137, 0.0295, 0.0093, 0.0798, 0.0612 , and 0.0589 , respectively.

$$
\begin{aligned}
\mathrm{IQ}= & -50.21+32.03 \mathrm{MD}+309.4 \mathrm{M}+0.364 \mathrm{NE} \\
& -0.62 \mathrm{UE}-1335 \mathrm{GD}+42526 \mathrm{SD}-18.1 \mathrm{SC} .
\end{aligned}
$$

The $p$ value for the IQ model is 0.0002 . The $R^{2}$ value for the model is 0.864 , which indicates that $86.4 \%$ of the variability of the IQ scores can be explained by the model. The IQ model also shows positive associations between IQ and MD (a high average number of direct connections between concepts may increase IQ), M (a highly clustered iWordNet may be associated with a high IQ), NE (more concepts and connections may increase IQ), and SD (adjusting for increases in connections due to experimental time differences) and negative associations between IQ and unique edges (adjusting for overcorrection of NE), graph density (a high level of connectedness between all concepts may indicate one's confusion), and stabilized centrality (adjusting for increases in connections due to experimental time differences). Figure 4 


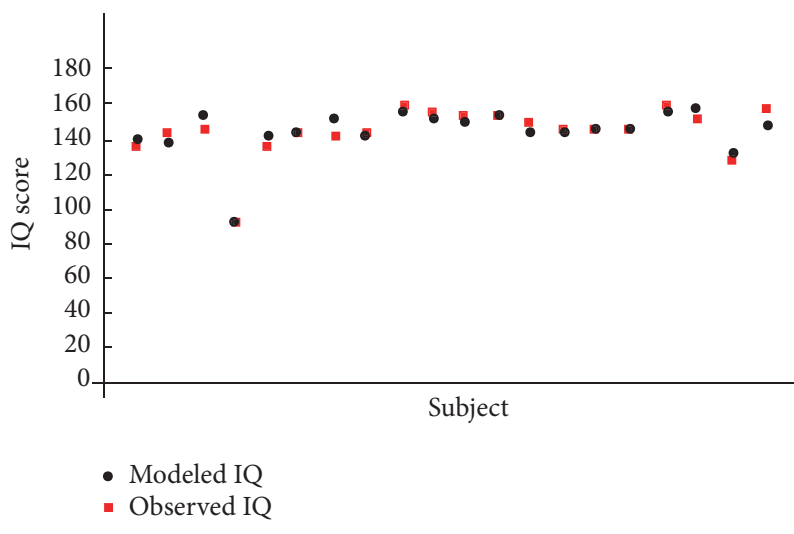

FIGURE 4: IQ modeling based on 20 iWordNets.

shows model predicted IQ scores (circles) versus the observed values (squares).

The left brain IQ (measuring memory, word comprehension, logic, etc.) model includes the variables mean geodesic distance, mean closeness centrality, total edges, unique edges, graph density, stabilized density, and stabilized centrality with associated $p$ values of $0.0129,0.0418,0.0780,0.0325$, $0.0083,0.0456$, and 0.0403 , respectively. The model $p$ value is 0.006 and $R^{2}$ is 0.838 . The left brain IQ model shows that IQ increases when mean closeness centrality, total edges, stabilized density, and stabilized centrality increase and mean geodesic, unique edges, and graph density decrease. The right brain IQ includes the variables modularity, total edges, vertices, graph density, stabilized density, and stabilized centrality, with associated $p$ values of $0.0622,0.0403,0.0170$, $0.0237,0.0452$, and 0.0316 , respectively. The model $p$ value is 0.0091 and $R^{2}$ is 0.686 . The right brain IQ (measuring perceptions, spatial relations, creativity, etc.) model shows that IQ increases when modularity, total number of edges, and stabilized density increase and vertices and stabilized centrality decrease.

These positive findings between IQ and iWordNets from this pilot study are very encouraging, although larger studies are necessary to confirm the findings of this general relationship (see Section 6), since the relationship between IQ and iWordNet topology is dependent on how the iWordNets are constructed. More importantly, larger studies are needed to explore the relationship between iWordNet topology and other aspects of cognitive development.

\section{Utilizing Local Topological Properties of the iWordNet}

5.1. The Path of Understanding. The global topology of an iWordNet can measure the overall knowledge of an individual as well as his knowledge and intelligence in different fields. However, the global topology is insufficient in describing an individual's understanding of each concept (word, phrase, sentence, etc.). Local topological properties of an iWordNet have to be used to describe an individual's understanding of particular concepts, as elaborated below.
According to distributional similarity or distributional semantics in computational linguistics, the meaning of a word is associated with its location in the sentence or the distribution of surrounding words $[42,43]$. If the word "foot" wherever and whenever it appears was replaced with the word "hand," and vice versa, the meanings of "foot" and "hand" would be switched. That is why different languages in the world almost work equally well in their own environments. The concept of distribution semantics is further developed using iWordNets: the meaning of a word in distributional semantics can be viewed as the aggregated meaning, which is individualized through the iWordNet. This implies that the meaning of a word is an individualized understanding and one shifts as one's iWordNet evolves. However, the meanings or topological properties of isolated words cannot carry us very far. We have to study how a concept string is expressed in an iWordNet. To this end, we introduce the concept of the "Path of Understanding" and describe its numerization.

An individual's understanding of a concept or a string of words (a phrase, sentence, paragraph, etc.) is determined by the path in the iWordNet marked by the sequence of the words in the word string, called the Path of Understanding (PoU). The sequence of topological properties (such as degree, centrality, and geodesic distance) of the nodes along the path forms a sequence of vectors. We call such a sequence of numerical vectors a numerization string. This numerization string characterizes an individual's understanding of the word string.

Let us use the sentence "We can use a network approach to study the mind" to illustrate the method: suppose that from a person's iWordNet, the words "we," "can," "use," "a," "network," "approach," "to," "study," "the," and "mind" have degrees $5,4,7,3,2,9,12,6,3$, and 8 , respectively. The numerization string $\{5,4,7,3,2,9,12,6,3,8\}$ is a numerical representation of the person's understanding of the sentence. Of course, different people have different understandings of the sentence, so their iWordNets and, therefore, numerization strings will differ.

If we want to characterize a person's understanding of word string more precisely, we can use more than one of the iWordNet's local properties, for example, degrees and centrality of vertices, on the PoU. Suppose that in our example above, in addition to the words' degrees, the centralities of "we," "can," "use," "a," "network," "approach," "to," "study," "the," and "mind" are 0.001, 0.003, 0.0012, 0.0023, 0.0015, $0.0032,0.004,0.0012,0.003$, and 0.0026 , respectively. The numerization string becomes $\{(5,0.001),(4,0.003),(7,0.0012)$, $(3,0.0023),(2,0.0015),(9,0.0032),(12,0.004),(6,0.0012),(3$, $0.003),(8,0.0026)\}$. Similarly, we can construct numerization strings with three or more different properties of iWordNets for more precise characterizations. It is interesting to note that numerization strings can also be visualized or converted to sound or music. Perhaps, most significantly, the numerization string provides a foundation for individualized statistical learning, such as Bayesian learning tools, artificial neural networks, and deep learning models.

5.2. Acquiring New Knowledge. How does a person acquire new knowledge or a new word? We can explain this using a 


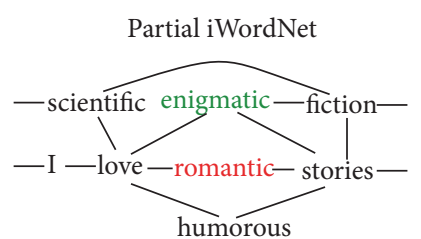

FIGURE 5: Illustration of smoothing on the Path of Understanding (PoU).

smoothing method over the iWordNet. The idea of smoothing is similar to the so-called smoothing method in modern control theory. If a word is missing in a sentence, we often use the smoothing technique to guess the meaning of the word in a way that makes sense in the sentence. Similarly, suppose one reads the sentence: "I love romantic stories." If the word "romantic" is new to him, he would make a guess about its meaning such that the sentence makes sense to him (smoothing). The meaning guessed must be closely related to the two words (love and stories) immediately before and after "romantic." We use the shortest distance (geodesic distance) to reflect the closeness and maximum number of links (degree) to reflect the commonness. In case there are multiple words, the maximization of distributional similarity or cosine similarity [44] and various statistical learning tools can also be used to understand the subject. With the assistance of the numerization of strings, smoothing using neighboring sentences can become much easier.

If a person reads the sentence "I love romantic stories" and does not recognize "romantic," he may use "enigmatic" to guess the meaning. Since "romantic" is not in his iWordNet, the two geodesics between love and stories, corresponding to the words "enigmatic" (with a degree of 3) and "humorous" (with a degree of 3), can help him make the guess. From past experiences, the meaning of "romantic" is more likely to be closer to "enigmatic" (with probability 3/5) than to "humorous" (with probability 2/5).

The idea of new knowledge acquisition can be based on the maximization of cosine similarity and when cosine similarities are the same for several Paths of Understanding then the probability Degree/ $\sum$ Degree will be associated with

several possible meanings. In the example (Figure 5), the cosine similarity between "I love enigmatic stories" and "I love romantic stories" is the same as the cosine similarity between "I love humor stories" and "I love romantic stories," but the associated probabilities, $3 / 5$ versus $2 / 5$, respectively, are different.

Here we raise an interesting paradox, even if we will not attempt to resolve it: as Einstein [45] said, "Pure logical thinking cannot yield us any knowledge of the empirical world...." If people understand a new concept by means of old, existing concepts, then what is the essence of new knowledge learned?

\section{Limitations}

In the pilot study, we were not able to address many questions regarding iWordNet construction such as the following: should we limit construction to single or multiple starting words (we always started with "smart")? Should we treat different forms of a word as the same thing (different tenses)? Should we limit the time for constructing an iWordNet? Should we use directed or undirected and weighted or unweighted networks? Should we limit the depth of a question chain (i.e., geodesic distance)? Should we only use key words in an iWordNet and what constitutes a key word? Should we differentiate between iWordNets with a general versus specific purpose?

\section{Discussion}

The Paradox of Understanding (meaning is meaningless) led to the iWordNet formulated by recursive definitions. Consequently, the global topological properties of an iWordNet holds the information that represents an individual's overall knowledge, IQ, personality, or status of cognitive development, and the local properties and numerization of PoU hold the information of the understanding of particular concepts. Since an iWordNet is primarily a representation of one's knowledge, it makes perfect sense to study cognitive science and artificial intelligence through network science. Network topology might also have a far-reaching impact on cognitive capacities that support or influence learning [34]. Studying how topological properties of iWordNets and neural networks drive the process of human learning might bridge branches of cognitive science.

Unlike the traditional study of cognitive science and artificial intelligence, for which the meanings of words are essential, the iWordNet approach intends to identify relationships between the topological properties of the iWordNet and an individual's personality, behavior, and cognitive development. This may be difficult to accept at first glance because the topological properties appear to be irrelevant to the meaning of the words, but it is evidenced by (1) the meaning of a word depends on where and how it is used, making the location of a word relative to others important, (2) the recursive explanation of a word by other words (i.e., circular definitions) makes words merely placeholders (as we discussed in the section of connotation of understanding), and (3) the pilot study, though small, did show a statistically significant association between IQ and the topology of iWordNets. However, the significance of the results of the pilot study is not about how iWordNets can predict IQ, but how it enlightens our appreciation of iWordNets as a new way of studying cognitive science.

We want to emphasize that understanding has to be on an individual basis. Even when we use the same dictionary for the definition of a word, the meaning of the word can only make sense through an individual Path of Understanding in the brain or iWordNet. Thus, it still circles back to individualbased understanding. The Path of Understanding (PoU) in an iWordNet and its numerization provide a tool for quantitative studies of meaning or individual understandings of a word, concept, sentence, or passage. The topological properties and numerization of the PoU technique can be used in clinics or experimental settings to study a person's cognitive aspects.

Just like a neurologist can obtain images of a patient's brain and determine where the damage is, we can construct 
an iWordNet to see the topological "damage" to a patient with Alzheimer's disease or a person who is mathematically impaired, for example. Thus, the potential treatments can be to directly target one's iWordNet or individualize teaching/learning methods to improve the "damage." The key to the success of the iWordNet approach is how to construct a reliable and sensible iWordNet, which is to be developed. To get a glimpse of the broad potential applications of iWordNet, the following is a list of examples:

(1) Evaluating one's knowledge over time using the iWordNet

(2) Evaluating one's personality over time using the iWordNet

(3) Analyzing and evaluating the cognitive impairment in patients or the elderly using the iWordNet

(4) Studying why people have different skills using the iWordNet

(5) Comparing the knowledge of different individuals using the iWordNet for improving teaching and learning

(6) Using the topologies of the iWordNet with other variables such as race, gender, and age to study the differences among different ethnic, gender, and age groups

(7) Studying people with communication deficiencies through the iWordNet

(8) Using the iWordNet combined with neurological and psychological approaches in research or in clinics

(9) Studying the process of new knowledge acquisition

(10) Making robots that truly understand humans, or, at least, using words and concepts in the same ways that humans do

(11) Studying organizational behaviors when the iWordNet is constructed using collective intelligence from an organization or specific group of people.

\section{Conclusion}

In the paper, we propose a new approach to study individualbased understanding. In this approach, we argue the meaning or understanding of a concept should be individualbased through the so-called understanding paradox, while the meaning of a concept by conventional wisdom is the collective individual understandings of a concept from the society. We proposed a simple method to construct iWordNet that connects different concepts of the same individual and postulate that such an iWordNet is a knowledge representation of the individual and thus, the topological properties of the iWordNet will characterize the person's knowledge, IQ, and so forth. We conducted a pilot study to prove that. The significance of the research work is that it could potentially open a new way to study artificial intelligence since one of the important aspects of AI is to study agent's understanding and knowledge. We further proposed the concept of "Path of Understanding" in an iWordNet that characterizes an individual's understanding of a complex concept such as a written passage. However, further discussions would exceed the scope of this paper. We hope this paper as an introductory, a proof-of-concept article, will attract more researchers in this area.

\section{Conflicts of Interest}

The authors declare that they have no conflicts of interest.

\section{Acknowledgments}

Thanks are due to Robert Piece for his proofreading and to Aleesha Ye for helping to collect data in the pilot study.

\section{References}

[1] A. R. Romberg and J. R. Saffran, "Statistical learning and language acquisition," Wiley Interdisciplinary Reviews: Cognitive Science, vol. 1, no. 6, pp. 906-914, 2010.

[2] R. N. Aslin and E. L. Newport, "Statistical learning: from acquiring specific items to forming general rules," Current Directions in Psychological Science, vol. 21, no. 3, pp. 170-176, 2012.

[3] R. N. Aslin and E. L. Newport, "Distributional language learning: Mechanisms and models of category formation," Language Learning, vol. 64, no. 2, pp. 86-105, 2014.

[4] A. Schapiro and N. Turk-Browne, "Statistical learning," in Brain Mapping: An Encyclopedic Reference, A. W. Toga, Ed., vol. 3, pp. 501-506, Elsevier, 2015.

[5] E. K. Johnson and M. D. Tyler, "Testing the limits of statistical learning for word segmentation," Developmental Science, vol. 13, no. 2, pp. 339-345, 2010.

[6] D. R. Ladd, S. G. Roberts, and D. Dediu, "Correlational studies in typological and historical linguistics," Annual Review of Linguistics, vol. 1, no. 1, pp. 221-241, 2015.

[7] A. L. Barabási, Network Science, Cambridge University Press, 2016.

[8] E. D. Kolaczyk, Springer: Statistical Analysis of Network Data: Methods and Models, Springer Science and Business Media, New York, NY, USA, 2009.

[9] T. G. Lewis, Network Science: Theory and Applications, Wiley, Hoboken, NJ, USA, 1 edition, 2009.

[10] P. Blackburn and J. Bos, "Representation and Inference for Natural Language: A First Course," in Computational Semantics (Studies in Computational Linguistics), Center for the Study of Language and Information, 2005.

[11] A. Clark and F. Fox, The Handbook of Computational Linguistics and Natural Language Processing, Wiley-Blackwell, 2012.

[12] T. Engelthaler and T. T. Hills, "Feature biases in early word learning: network distinctiveness predicts age of acquisition," Cognitive Science, vol. 41, pp. 120-140, 2017.

[13] A. E. Motter, A. P. S. de Moura, Y.-C. Lai, and P. Dasgupta, Topology of the conceptual network of language, vol. 65, URL http, 2002, http://dx.doi.org/10.1103/PhysRevE.65.065102.

[14] M. Steyvers and J. B. Tenenbaum, "The large-scale structure of semantic networks: statistical analyses and a model of semantic growth," Cognitive Science, vol. 29, no. 1, pp. 41-78, 2005.

[15] M. S. Vitevitch, K. Y. Chan, and R. Goldstein, "Insights into failed lexical retrieval from network science," Cognitive Psychology, vol. 68, no. 1, pp. 1-32, 2014. 
[16] J. Borge-Holthoefer and A. Arenas, "Semantic networks: Structure and dynamics," Entropy, vol. 12, no. 5, pp. 1264-1302, 2010.

[17] J. n. Goñi, I. Martincorena, B. Corominas-Murtra, G. Arrondo, S. Ardanza-Trevijano, and P. Villoslada, "Switcher-randomwalks: a cognitive-inspired mechanism for network exploration," International Journal of Bifurcation and Chaos, vol. 20, no. 3, pp. 913-922, 2010.

[18] R. F. I. Cancho, R. V. Solé, and R. Köhler, "Patterns in syntactic dependency networks," Physical Review E, vol. 69, no. 5, Article ID 051915, 2004.

[19] E. R. Chrastil and W. H. Warren, "Active and passive spatial learning in human navigation: Acquisition of graph knowledge," Journal of Experimental Psychology: Learning Memory and Cognition, vol. 41, no. 4, pp. 1162-1178, 2015.

[20] D. Papo, J. M. Buldu, S. Boccaletti, and E. T. Bullmore, "Complex network theory and the brain," Philosophical Transactions of the Royal Society B: Biological Sciences, vol. 369, no. 1653, pp. 20130520-20130520, 2014.

[21] D. Goldenberg and A. Galván, "The use of functional and effective connectivity techniques to understand the developing brain," Developmental Cognitive Neuroscience, vol. 12, article no. 265, pp. 155-164, 2015.

[22] J. D. Medaglia, M.-E. Lynall, and D. S. Bassett, "Cognitive network neuroscience," Journal of Cognitive Neuroscience, vol. 27, no. 8, pp. 1471-1491, 2015.

[23] O. Sporns and R. F. Betzel, "Modular brain networks," Annual Review of Psychology, vol. 67, pp. 613-640, 2016.

[24] A. R. Backes, D. Casanova, and O. M. Bruno, "A complex network-based approach for boundary shape analysis," Pattern Recognition, vol. 42, no. 1, pp. 54-67, 2009.

[25] A. R. Backes and O. M. Bruno, "Shape classification using complex network and multi-scale fractal dimension," Pattern Recognition Letters, vol. 31, no. 1, pp. 44-51, 2010.

[26] A. R. Backes, D. Casanova, and O. M. Bruno, "Texture analysis and classification: A complex network-based approach," Information Sciences, vol. 219, pp. 168-180, 2013.

[27] J. Cong and H. Liu, "Approaching human language with complex networks," Physics of Life Reviews, vol. 11, no. 4, pp. 598-618, 2014.

[28] N. M. Beckage and E. Colunga, "Language networks as models of cognition: Understanding cognition through language," Towards a Theoretical Framework for Analyzing Complex Linguistic Networks, pp. 3-28, 2015.

[29] V. Nastase, R. Mihalcea, and D. R. Radev, "A survey of graphs in natural language processing," Natural Language Engineering, vol. 21, no. 5, pp. 665-698, 2015.

[30] C. S. Q. Siew, "Community structure in the phonological network," Frontiers in Psychology, vol. 4, Article ID Article 553, 2013.

[31] P. Gravino, V. D. P. Servedio, A. Barrat, and V. Loreto, "Complex structures and semantics in free word association," Advances in Complex Systems, vol. 15, no. 3-4, Article ID 1250054, 2012.

[32] A. Utsumi, "A complex network approach to distributional semantic models," PLoS ONE, vol. 10, no. 8, Article ID 0136277 , 2015.

[33] B. Corominas-Murtra, S. Valverde, and R. SolÉ, “The ontogeny of scale-free syntax networks: Phase transitions in early language acquisition," Advances in Complex Systems, vol. 12, no. 3 , pp. 371-392, 2009.

[34] E. A. Karuza et al., "Local patterns to global architectures," in Influences of Network Topology on Human Learning Trends in Cognitive Sciences, vol. 20, 8 edition, August 2016.
[35] D. R. Radev, M. T. Joseph, B. Gibson, and P. Muthukrishnan, "A bibliometric and network analysis of the field of computational linguistics," Journal of the Association for Information Science and Technology, vol. 67, no. 3, pp. 683-706, 2016.

[36] Z. Wang, C. Xia, S. Meloni, C. Zhou, and Y. Moreno, "Impact of Social Punishment on Cooperative Behavior in Complex Networks," Scientific Reports, vol. 3, no. 1, 2013.

[37] F. Zhang et al., "Community detection based on links and node features in social networks," in Proceeding of the MultiMedia Modeling: 21st International Conference, Part I, I. He and T., Eds., pp. 418-429, Springer International Publishing, Switzerland, 2015.

[38] H. Matsuno, Y. Tanaka, H. Aoshima, A. Doi, M. Matsui, and S. Miyano, "Biopathways representation and simulation on hybrid functional Petri Net," In Silico Biology, vol. 3, no. 3, pp. 389-404, 2003.

[39] I. Koch, B. H. Junker, and M. Heiner, "Application of Petri net theory for modelling and validation of the sucrose breakdown pathway in the potato tuber," Bioinformatics, vol. 21, no. 7, pp. 1219-1226, 2005.

[40] M. Chang, Monte Carol Simulations for the Pharmaceutical Industry, CRC Press, Boca Raton, FL, USA, 2010.

[41] D. Hansen, B. Shneiderman, and M. A. Smith, Analyzing Social Media Networks with NodeXL: Insights from a Connected World, Morgan Kaufmann, Burlington, Mass, USA, 1st edition, 2010.

[42] H. Rubenstein and J. Goodenough, "Contextual correlates of synonymy," Communications of the ACM, vol. 8, no. 10, pp. 627633, 1965.

[43] P. Pantel, "Inducing ontological co-occurrence vectors," in Proceedings of the 43rd Annual Meeting of the Association for Computational Linguistics (ACL '05), pp. 125-132, Association for Computational Linguistics, Morristown, NJ, USA, June 2005.

[44] E. Agirre, E. Alfonseca, K. Hall, J. Kravalova, M. Paşca, and A. Soroa, "A Study on Similarity and Relatedness Using Distributional and WordNet-based Approaches, Human Language Technologies," in Proceedings of The 2009 Annual Conference of the North American Chapter of the ACL, pp. 19-27, Association for Computational Linguistics, Boulder, Colorado, May 2009.

[45] A. Einstein, "Ideas and opinions," Broadway Books; Reprint edition, June 6, 1995. 

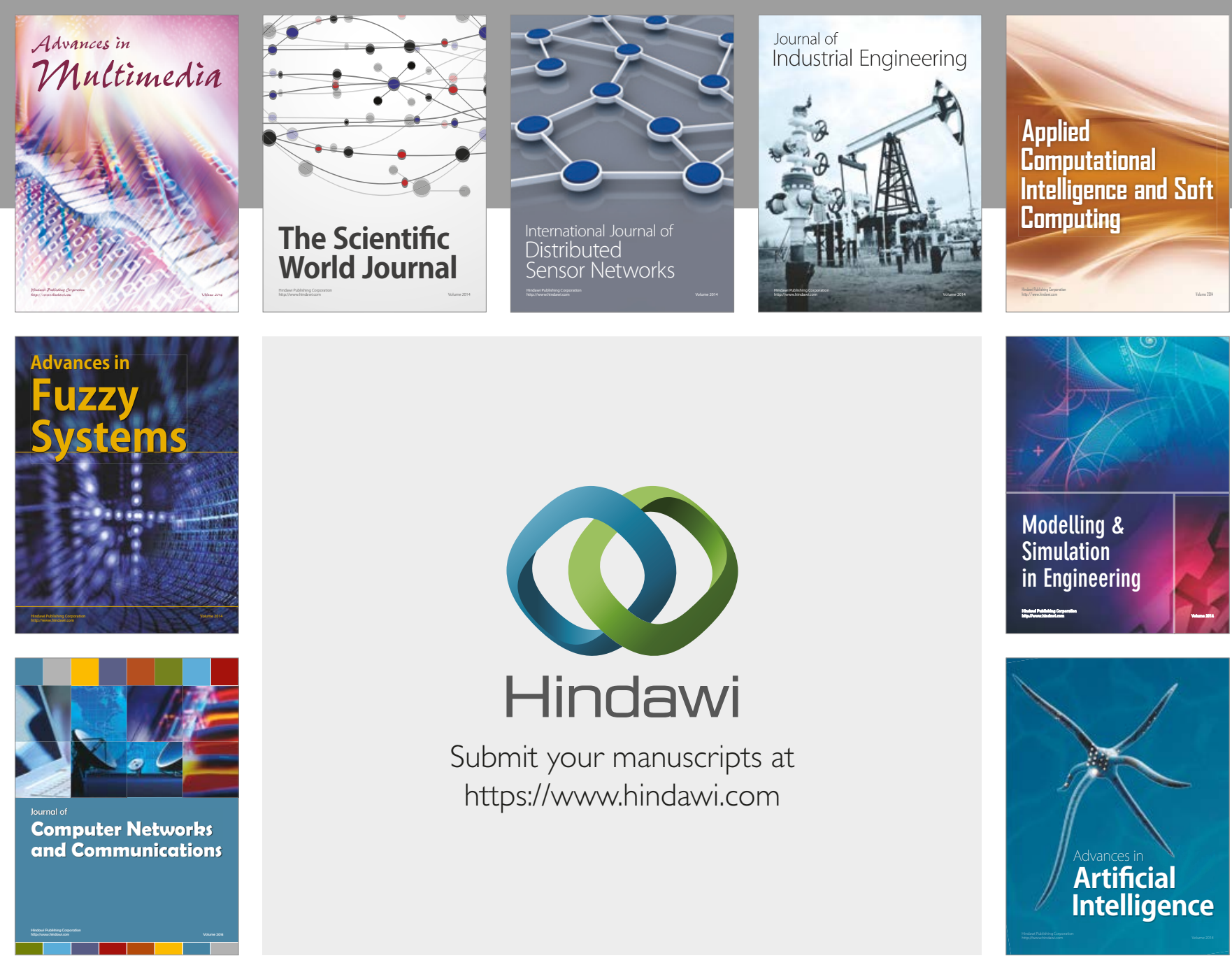

\section{Hindawi}

Submit your manuscripts at

https://www.hindawi.com
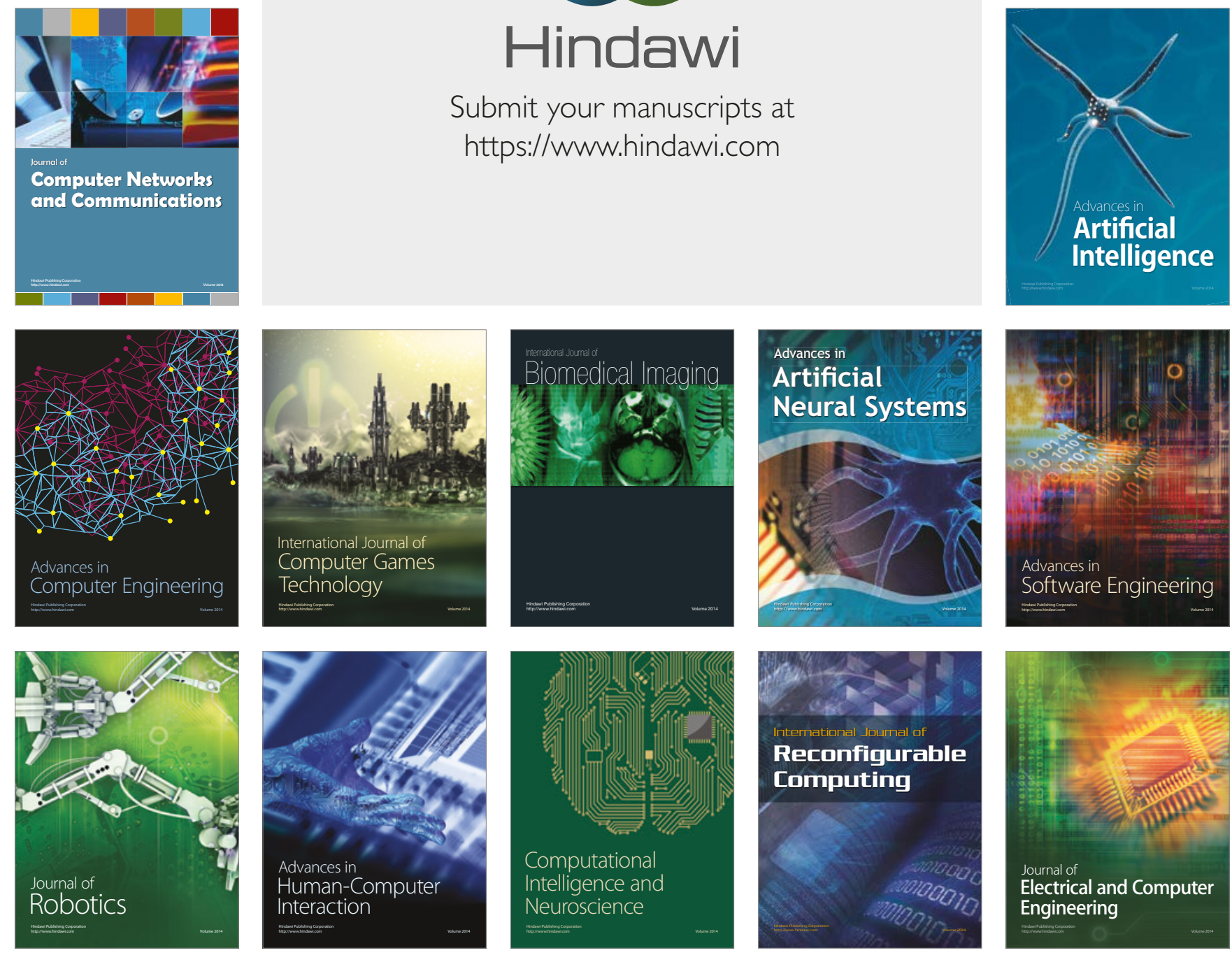